\title{
Discriminative stimulus control in instrumental electrodermal conditioning in the monkey (Cebus albifrons)
}

\author{
H. D. KIMMEL and A. F. BRENNAN \\ University of South Florida, Tampa, Florida
}

\begin{abstract}
Four Cebus albifrons monkeys received 24 sessions of multiple avoidance-punishment conditioning of the skin conductance response (SCR) with no discriminative stimuli, followed by 24 additional sessions with visual discriminative stimuli identifying the avoidance and punishment components. Four other monkeys that had previously been conditioned with the discriminative stimuli were given 24 additional sessions without them. Yoked controls were run with this group and received the electric shock noncontingently. The presence of the discriminative stimuli clearly facilitated acquisition and maintenance of higher SCR frequencies in avoidance as compared with punishment. Acquisition was retarded with the stimuli absent, and higher overall response levels resulted. Adding the stimuli improved differentiation and resulted in lower overall rates. Removing the stimuli likewise resulted in higher response frequency. When the stimuli were absent initially, higher shock frequencies were administered, and when they were removed, shock frequency increased. The yoked controls increased in response frequency but did not differentiate between the avoidance and punishment conditions. Overall, the presence of the stimuli appeared to facilitate differentiation and reduce trauma, as compared with when they were absent.
\end{abstract}

Evidence that subhuman primates (Cebus albifrons) learn to differentiate in terms of electrodermal response frequency between visual discriminative stimuli that signal either shock-avoidance or responsecontingent punishment has recently been reported by Kimmel, Brennan, McLeod, Raich, and Schonfeld (1979). Their monkeys received 25 conditioning sessions with red and green visual stimuli that identified periods of shock-postponement contingent upon unelicited skin conductance responses (SCR) and periods of shock-punishment of SCRs. Beginning with the third conditioning session and continuing thereafter, significantly higher SCR frequencies were observed during avoidance than during punishment, providing evidence of instrumental autonomic conditioning in the monkey. This difference was also present in an extinction session 3 months after acquisition.

The purpose of the present study was to examine in greater detail the role of discriminative stimuli in the acquisition and maintenance of discriminative instrumental SCR conditioning in the monkey. Specifically, a new group of four Cebus albifrons monkeys initially received essentially the same condition-

This research was funded by Contract 17-76-6053, U.S. Army Medical Research and Development Command. The data reported here represent a portion of the MA thesis submitted by the second author to the University of South Florida. A preliminary report on this work was presented to the Psychonomic Society in November 1979. H. D. Kimmel's mailing address is: Department of Psychology, University of South Florida, Tampa, Florida 33620. ing procedures as those previously used, except that no visual discriminative stimuli were administered during the avoidance and punishment periods. Following these 24 acquisition sessions without the visual discriminative stimuli, the monkeys then received 24 additional conditioning sessions with the stimuli added. For comparison purposes, four monkeys selected at random from the Kimmel et al. (1979) study were given refresher conditioning sessions with their original discriminative stimuli (to overcome the extinction they had received 6 months earlier, at the end of the previous study), and then were given 24 conditioning sessions without the stimuli. Another four monkeys, previously used in a classical conditioning study (Kimmel \& Burns, 1975), were run as yoked controls to the monkeys shifted to no discriminative stimuli. Thus, the present report concerns one group of monkeys conditioned initially without discriminative stimuli and then shifted to conditioning with the stimuli added, a second group of monkeys given the reverse arrangement, and a third group yoked to the second group.

It was expected that differentiation in SCR frequency between the avoidance and punishment periods would be greatly reduced or retarded when the reinforcement contingencies were administered initially without the discriminative stimuli. The addition of the discriminative stimuli following training without them was expected to enhance whatever differentiation was already present, or to enable differentiation to be achieved if it had not been previously. 
No prediction could be made regarding the effect of removing the discriminative stimuli following conditioning with them, except that the existing differentiation between avoidance and punishment was likely to deteriorate. The stimulus-removal procedure was administered primarily to provide a control comparison with the stimuli absent to aid in the interpretation of the effect of initial conditioning without the stimuli. The yoked control group was run to determine whether the pattern of shocks received in avoidance and punishment would generate differences in SCR frequency resembling those obtained in an instrumental condition.

\section{METHOD}

\section{Subjects}

Twelve adult male Cebus albifrons monkeys served as subjects in this study; eight of them had been used previously (Kimmel et al., 1979; Kimmel \& Burns, 1975) and four were experimentally najve. The four new animals, referred to as the discriminative stimulus added (DSA) group, were first habituated for 2 weeks to handling and sitting in the primate chair. Then 2 additional weeks of baseline data collection were administered to these animals before the introduction of the conditioning procedures. Four of the previously used animals, referred to as the discriminative stimuli removed (DSR) group, were given four refresher conditioning sessions prior to the implementation of the new conditioning procedures with the stimuli removed. These refresher sessions were run under the same condition as had previously been used during the original conditioning with these monkeys and using the same discriminative stimuli. The purpose of the refresher conditioning sessions was to restore the original conditioning that had existed prior to the administration of two extinction sessions 6 months before the present study. The yoked controls had been run more than 1 year previously in a classical conditioning study with tones and shocks, followed by extinction. These animals were run to assess the influence of noncontingent shocks on SCR frequency.

\section{Apparatus}

The apparatus and stimulus parameters were identical to those used by Kimmel et al. (1979), except as noted below. The monkey was restrained in a primate chair with SCR electrodes attached to the plantar surfaces of both feet, shock electrodes attached to the tail, and two stress-test cardiac electrodes (K\& G, DME 95-40 Pediatric Foam)' ${ }^{\prime}$ on the upper chest. Heart rate was amplified and digitized for input to the computer by relay-closure coincident with the $\mathbf{R}$ wave of each heartbeat. This permitted counting of heartbeats and timing of interbeat intervals to be done automatically by the computer.

The digitizing of the SCR utilized time bins of $.33 \mathrm{sec}$ and an amplitude criterion of .18 micromhos, increased from the .13 micromhos previously used. This change was introduced to increase the reliability of scored responses. Criterion increases in SCR amplitude across two successive time bins were required for a response to be counted, as previously. The criterion for computer detection of sudden downward shifts in skin conductance, which occur during vigorous movements, was increased from the previous .4 micromhos to .6 micromhos, in time bins increased from .1 sec to $.33 \mathrm{sec}$, again to increase reliability in this procedure. As previously, to prevent "rebound" conductance increases from being counted as responses and reinforced or punished, these sudden decreases in conductance were followed by $2.0 \mathrm{sec}$ of time-out from contingencies.

\section{Procedure}

Conditioning procedures were identical to those used previously in the Kimmel et al. (1979) study, except as noted. The duration of the avoidance and punishment segments varied randomly among 3.16, 4.16, and $4.66 \mathrm{~min}$, with a mean of $4.00 \mathrm{~min}$. Four avoidance and punishment segments were administered in each session, in a random sequence with no more than two successive segments of the same type. Adjacent segments were separated by 30 -sec periods of time-out from contingencies. In addition, there was a 30-sec period at the beginning of each segment (whether or not the discriminative stimuli were present) without contingencies, to permit observation of dependent measures in the absence of the contingencies. This period was lengthened to $90 \mathrm{sec}$ at the beginning of the last segment of each session, to increase the likelihood that existing avoidance-punishment differences could be observed during these periods. This lengthened segment could be either an avoidance or a punishment segment, depending on the random programming of segment sequences.

The DSA group received 24 conditioning sessions, 2 per week, without discriminative stimuli (initial training), followed by 24 conditioning sessions with the stimuli added (secondary training). Since the monkeys in the DSR group had received their initial training with the stimuli present during the previous study, they were given secondary training consisting of 24 conditioning sessions with the stimuli removed. The yoked control simply received shocks whenever the DSR monkey did.

\section{RESULTS}

\section{Frequency of Instrumental SCRs}

Unelicited SCRs were measured during administration of the avoidance and punishment contingencies and converted to frequency of $\mathrm{SCR} / \mathrm{min}$. Figure 1 shows the average frequency of $\mathrm{SCR} / \mathrm{min}$ in sixsession blocks for the DSA group during the four blocks of initial and four blocks of secondary training. For comparison purposes, Figure 1 also shows the SCR/min data from the first and last blocks of six sessions of initial and secondary training for the DSR animals. It should be noted that the first and fourth blocks in initial training data for the DSR animals were reported previously in Kimmel et al. (1979). Only the first and last blocks of secondary

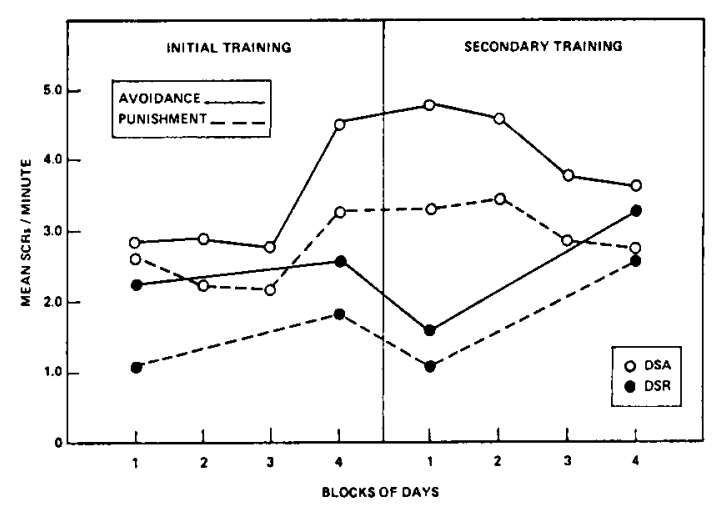

Figure 1. mean avoidance and punishment frequencies of SCR/ min during Blocks 1 through 4 of initial and secondary training in DSA animals and during the first and fourth blocks in initial and secondary training in DSR animals. 
training data are given here to maintain consistency with the earlier presentation and to permit straightforward analyses involving both initial and secondary training for the DSR animals. ${ }^{2}$

Analysis of variance of $\mathrm{SCR} / \mathrm{min}$ in the DSA group showed that the overall avoidance-punishment difference was significant $[F(1,3)=19.46, p<.05]$ and that the three-way interaction of initial-secondary training, blocks, and avoidance-punishment was significant $[F(3,9)=8.92, p<.01]$. As can be seen in Figure 1, this three-way interaction reflected the fact that the avoidance-punishment difference in the DSA group increased across the four blocks in initial training but was maintained at a more or less stable level over blocks during secondary training. Separate postmortem $t$ tests indicated that the avoidance-punishment difference attained significance in the second block in initial training and remained significant thereafter (all ps $<.01$ ). The avoidance $>$ punishment difference was not significant in any single session of the first block of initial training. Indeed, these animals actually made more responses during punishment than avoidance in each of the first three daily sessions. The avoidance $>$ punishment difference did not achieve significance in the DSA animals until the eighth session of initial training. In contrast, this difference became significant in the third daily session of initial training in the DSR group (Kimmel et al., 1979).

The interaction of initial-secondary training and blocks was also significant in the DSA group's SCR/ min data $[F(3,9)=11.08, p<.01]$. This interaction is a manifestation of the fact that overall SCR frequency (avoidance and punishment combined) increased in the DSA group across blocks during initial training, which was administered without the discriminative stimuli, but decreased across blocks during secondary training, with the stimuli added. Trend analysis showed that this reversal was primarily linear, since the linear component of the initial-secondary training $\times$ blocks interaction was significant $[F(1,9)=25.76, p<.05]$, while the quadratic and cubic components of the interaction did not approach significance.

Kimmel et al. (1979) reported that the overall avoidance-punishment difference in the DSR group's $\mathrm{SCR} / \mathrm{min}$ data during initial training was significant. When the DSR animals were shifted to further conditioning with the discriminative stimuli removed, there was a significant reduction in this avoidancepunishment difference $[F(1,3)=24.71, p<.01]$. No other effects were significant in this separate analysis of the SCR frequency data in the DSR group.

Using data from only the first and fourth blocks in initial and secondary training of both DSA and DSR groups, a combined analysis of variance showed that the overall avoidance-punishment effect was sig- nificant $[F(1,6)=31.46, p<.01]$. The three-way interaction of groups (DSA vs. DSR), avoidancepunishment, and initial-secondary training was also significant in this analysis $[F(1,6)=6.27, p<.05]$, since the DSA group showed greater avoidancepunishment differentiation during secondary training (with the discriminative stimuli added) than during initial training (without the stimuli), while the DSR group showed greater avoidance-punishment differentiation during initial training (with the stimuli) than during secondary training (with the stimuli removed). In addition, avoidance-punishment differentiation came into existence and was maintained across blocks differently in the two groups and in initial and secondary training; thus, a significant four-way interaction also was obtained in this analysis $[F(1,6)=21.57, p<.01]$.

A separate analysis of variance was conducted on the SCR/min data of the DSA and the DSR groups during initial training only, again using only the first and fourth blocks. The overall avoidancepunishment difference was significant $[F(1,6)=22.18$, $\mathrm{p}<.01$, as was the overall increase in frequency of SCR/min from the first to the fourth block $[F(1,6)$ $=10.94, \mathrm{p}<.05]$. This analysis also showed that the three-way interaction of groups, avoidancepunishment, and blocks was significant $[F(1,6)=$ $15.51, p<.05]$, since the avoidance-punishment difference increased substantially from the first to the fourth block in the DSA group (being all but nonexistent in the first block) but was present equally in the first and fourth blocks in the DSR group.

Figure 2 presents the average SCR frequency in four blocks of secondary training for the DSR animals, along with comparable data from their yoked controls. Analysis of variance of these data showed that the overall increase over blocks and the overall avoidance $>$ punishment difference were both significant $[F(3,18)=6.20, p<.01$, and $F(1,6)=13.16$,

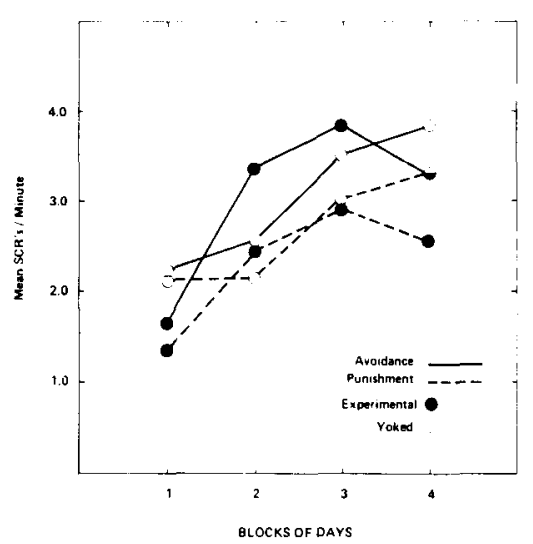

Figure 2. Mean avoidance and punishment frequencies of SCR/ min during four blocks of secondary training in DSR and yoked control animals. 
$p<.05$, respectively]. The overall increase in response frequency across sessions within blocks was also significant $[\mathrm{F}(3,18)=5.19, \mathrm{p}<.01]$. The interaction of avoidance vs. punishment, sessions, and groups was also significant in this analysis $[F(5,30)=$ $3.08, \mathrm{p}<.05$ ], since the avoidance $>$ punishment difference increased systematically over sessions in the DSR group but not in the yoked controls. This difference was statistically significant in the DSR group $(t=3.12, p<.025)$ but not in the yoked controls $(t=1.65)$.

\section{SCR Frequency During Initial \\ Contingency-Free Periods}

The computer was programmed to exclude the first $10 \mathrm{sec}$ of these initial periods and to count SCRs during the next $20 \mathrm{sec}$. The first $10 \mathrm{sec}$ were excluded to prevent counting as unelicited those responses that may have been elicited by the onset of the discriminative stimuli (when discriminative stimuli were present or at comparable times when stimuli were absent). In the DSA group, the frequency of these SCRs increased across blocks during initial training (with no stimuli) and declined across blocks during secondary training (with stimuli). This resulted in a significant interaction of initial-secondary training and blocks $[F(3,9)=12.86, p<.01]$. No avoidance-punishment effects were observed in these data. A combined analysis of variance using both groups revealed a significant interaction of initialsecondary training, blocks, and groups $[F(1,6)=$ $8.39, p<.05$ ], since the pattern of increase in initial training followed by decrease in secondary training, seen in the DSA group, was not present in the DSR group. In the DSR group, the frequency of $\mathrm{SCR} / \mathrm{min}$ during the contingency-free periods increased across blocks during both initial and secondary training. This also occurred in their yoked controls.

The expectation that avoidance-punishment or group differences might be observable during lengthened contingency-free periods at the beginning of the last segment of each session was not borne out by the data. Thus, even with lengthened contingencyfree periods, avoidance-punishment differences were present only during the administration of the contingencies and increased across sessions, except in the yoked controls.

\section{Heart Rate}

Two different kinds of evaluation of the heart rate (HR) data were conducted. First, HR during the 10 -sec periods immediately preceding the implementation of avoidance and punishment contingencies was reduced to beats/min and the mean HRs for avoidance and punishment were compared. These measures showed no avoidance-punishment differ-

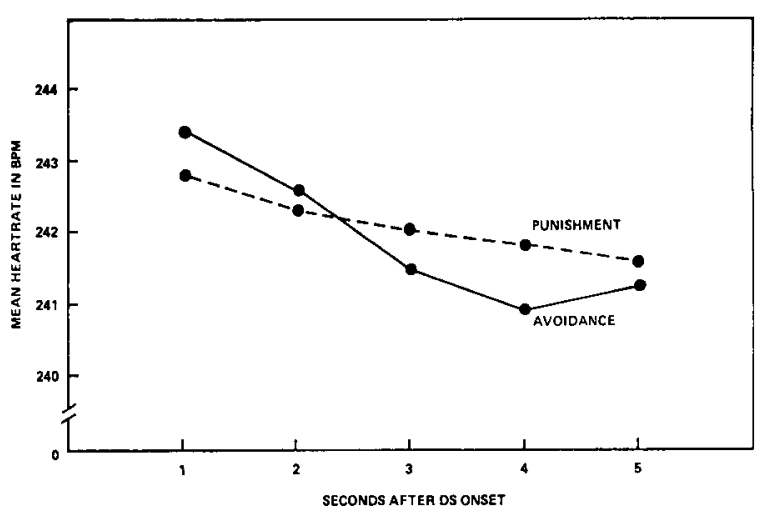

Figure 3. Mean heart rate during first 5 sec after discrimlnatlve stimulus onset in avoidance and punishment during secondary training in DSA animals.

ences, whether or not discriminative stimuli were present.

The second type of evaluation of the HR data was achieved by programming the computer to score the first 26 interbeat intervals following the onset of the discriminative stimulus. These interbeat intervals were converted to mean $H R$ in beats/min for each of the first $5 \mathrm{sec}$ following stimulus onset. These data could be analyzed only for the DSA animals during secondary training (i.e., when a discriminative stimulus was present). Figure 3 presents the course of HR change elicited by the onset of the discriminative stimulus, separately for avoidance and punishment segments. As shown in Figure 3, the elicited $H R$ change was decelerative under both contingencies $[F(4,12)=4.42, p<.05]$ and more pronounced in avoidance than in punishment $[F(4,12)=$ $7.54, \mathrm{p}<.011$. The decelerative $H R$ pattern increased in amount across the four blocks of secondary training sessions $[F(12,36)=3.82, p<.01]$, but this interaction (seconds $\times$ blocks) was not influenced by the avoidance-punishment variable.

\section{Number of Shocks}

Kimmel et al. (1979) reported that significantly more shocks occurred during punishment than during avoidance when discriminative stimuli were employed during conditioning. This difference was even greater during initial training in the DSA group in the present study, mainly because the DSA animals showed higher frequencies of SCR/min (cf. Figure 1). Analysis of variance of the DSA and DSR groups' shock frequencies during initial training showed that the two groups differed significantly in overall number of shocks received $[F(1,6)=9.57$, $\mathrm{p}<.05]$ and that their combined avoidance-punishment difference increased from the first to the fourth block of initial training $[F(1,6)=10.89, p<.05]$. The interaction of avoidance-punishment, blocks, segments, and groups was also significant $[F(3,18)=$ 
$3.75, \mathrm{p}<.05]$, reflecting the fact that the DSR group's avoidance-punishment difference increased across segments in the first block but decreased across segments in the fourth block, while the DSA group's avoidance-punishment difference was unchanged across segments in the first block but increased markedly across segments in the fourth block.

The addition of discriminative stimuli in secondary training in the DSA group resulted in an increase in shock frequency during avoidance and a decrease in shock frequency during punishment. Analysis of variance of number of shocks received by the DSA group during initial and secondary training revealed that the interaction between avoidance-punishment and initial-secondary training was significant $[F(1,3)$ $=11.15, \mathrm{p}<.05]$. The overall avoidance-punishment difference, of course, was significant in the DSA group $[F(1,3)=11.65, p<.05]$.

Removal of the discriminative stimuli in secondary training in the DSR group did not result in significant reduction of the avoidance-punishment difference in shock frequency, although there was an increase in total number of shocks received. Of course, the yoked controls received the identical shock schedule.

In general, total shock frequency tended to be higher when the discriminative stimuli were absent than when the stimuli were present, although the interaction of groups and initial-secondary training (collapsing avoidance and punishment) failed to attain significance $[F(1,6)=5.46, p<.10]$.

\section{DISCUSSION}

The presence of exteroceptive discriminative stimuli that signal occasions of Sidman avoidance or response-contingent punishment clearly facilitated the acquisition and maintenance of differential instrumental SCR responding under these contingencies. When the stimuli were present during initial training in the DSR group, the avoidance-punishment difference in SCR/min was observable within the first block of conditioning sessions (significant on Session 3 and thereafter), but this difference did not become significant when conditioning was administered without the stimuli to the DSA group until the second block of conditioning. Indeed, during initial training, the avoidance-punishment difference in $\mathrm{SCR} / \mathrm{min}$ reached its maximum in the fourth block in the DSA animals but was already maximal in the first block and remained stable thereafter in the DSR group, as shown by the significant interaction of groups, avoidance-punishment, and blocks. Examination of Figure 1 shows that it was not until the fourth block of initial training that the avoidance-punishment difference in SCR/min attained a level comparable to that already achieved in the DSR group in the first block of conditioning sessions.

Yet, it should be noted that SCR differentiation was attained during initial training in the DSA group even without exteroceptive discriminative stimuli, albeit more slowly than in the DSR group, trained initially with the stimuli present. It is assumed that the rather large difference in number of shocks received during avoidance and punishment by the DSA animals (over four times as many shocks during punishment than avoidance in the first block of sessions) provided a basis for this differentiation even in the absence of explicit exteroceptive discriminative stimuli. Thus, while the presence of discriminative stimuli facilitated the acquisition of SCR avoidance-punishment differentiation, this differentiation did not require the presence of exteroceptive stimuli.

Introduction of discriminative stimuli after initial training without them had the effect of improving differentiation between avoidance and punishment in the DSA group, while removing the stimuli after training with them did not substantially influence differentiation in the DSR group. In this connection, it should be noted that differences in shock frequency in the DSR group increased to about four to one (punishment $>$ avoidance) by the fourth block of initial training, so that an additional basis for differentiation from this source existed after the stimuli were removed. Furthermore, the DSR group responded significantly more frequently during avoidance than with punishment in two extinction sessions without shocks (Kimmel et al., 1979).

Perhaps equally noteworthy regarding the role played by the discriminative stimuli in the present experimental situation is the fact that avoidancepunishment differentiation in the DSA group was achieved at the cost of substantially increased overall levels of SCR frequency. As can be seen in Figure 1, maximal avoidance-punishment differentiation during initial training was attained by the DSA group in the fourth block of conditioning session at the same time that these monkey's average number of SCR $/ \mathrm{min}$ increased considerably in both avoidance and punishment. The significant interaction of initial-secondary training and blocks in the DSA group testifies to the fact that these animals showed an increase in overall SCR frequency during conditioning without the stimuli and a decrease during further conditioning with the stimuli added. A similar increase in overall response frequency was not observed during initial training in the DSR animals; some increase occurred during secondary training with the stimuli removed, but this increase was not significant. A similar difference between the DSA and DSR groups was observed in the frequency of $\mathrm{SCR} / \mathrm{min}$ during initial contingency-free periods. In 
these measures, there were no significant avoidancepunishment differences in either group, but response frequency increased in the DSA group during initial training without the stimuli and decreased with further training with the stimuli added; this reversal did not occur in the DSR group. In other words, one consequence of differentialavoidance-punishment SCR conditioning without discriminative stimuli was that overall response frequency was elevated. Since this increase in response frequency resulted in increasingly higher numbers of shocks during punishment as compared with avoidance, it may be that the eventual differentiation in SCR/min between avoidance and punishment was a result of this elevated responding. The presence of the stimuli, from this point of view, may have been to permit avoidancepunishment differentiation to be achieved at less cost to the animal, in terms of total number of responses emitted.

The only avoidance-punishment difference observed in the HR data occurred in the DSA group during secondary training. As can be seen in Figure 3, the $\mathrm{HR}$ response to the onset of the discriminative stimulus was significantly more decelerative in avoidance than in punishment, but this may have resulted from greater classical defensive conditioning due to higher shock frequencies during punishment, resulting in more unambiguous orienting cardiac orienting reactions (i.e., deceleration) in avoidance. This elicited HR difference is similar to an effect noted by Kimmel et al. (1979) in the SCR elicited by the onset of the discriminative stimuli during extinction. The onset of the punishment discriminative stimulus elicited a significantly larger SCR than did the avoidance discriminative stimulus, probably because of the large difference in number of shocks during conditioning (punishment $>$ avoidance).

Multiple avoidance-punishment SCR conditioning without discriminative stimuli resulted in the reception of significantly greater numbers of shocks than did initial conditioning with the discriminative stimuli. This was probably a consequence of the elevated overall $\mathrm{SCR} / \mathrm{min}$ shown in the DSA group during initial training, as compared with the DSR group. Although the DSR group received increasingly more shocks during punishment than during avoidance across successive segments within the first block of conditioning sessions, this avoidance-punishment difference in shock frequency reduced across segments during the fourth block of sessions. In the DSA group, on the other hand, the already large avoidance-punishment difference in shock frequency that was present during the first block of conditioning sessions increased substantially across segments during the fourth block at the same time that the overall frequency of SCR/min increased markedly and the avoidance-punishment difference in SCR/ min became comparable to that shown throughout initial training by the DSR group. When the stimuli were added during secondary training, the DSA animals continued to receive relatively high frequencies of shock, although their overall SCR frequency reduced somewhat. When the discriminative stimuli were removed in secondary training, the DSR animals showed some increase in response frequency and, especially during the last block of secondary training, an increase in number of shocks received. Thus, the interaction of groups and initial-secondary training approached significance in the shockfrequency data.

The difference between avoidance and punishment in shock frequency, resulting in a difference in elicited HR changes (and in elicited SCRs in our previous study), suggests that shock frequency, as such, is not the basis of the observed differences in SCR frequency, favoring avoidance over punishment. If anything, higher shock frequency should lead to higher rather than lower SCR frequencies. As was seen in the present yoked controls, simply receiving different numbers of shocks does not result in significant (or even substantial) avoidance-punishment differentiation. As suggested above, differences in shock frequency during avoidance and punishment may provide a stimulus basis for acquiring the instrumental differentiation when no visual discriminative stimuli are available. But, since this shock-frequency difference itself results from differential response frequencies, some conditioning must occur even without large differences in shock frequency. It is likely that the shocks automatically programmed in avoidance cause an elevation in arousal that leads to responding during punishment. These responses, of course, result in additional shocks. But further elevation of nondifferential responding is reinforced via avoidance and, at this point, the instrumental differentiation becomes available.

It should be noted that the present multiple avoidance-punishment is not so simple that the monkey can easily learn skeletal response differentiation. Raich and Kimmel (1979) administered a similar multiple avoidance-punishment schedule to two monkeys, requiring a panel-press instead of the SCR. Both monkeys learned to respond significantly more frequently during avoidance than during punishment, but one monkey did this by suppressing responding drastically, while the other responded at a high rate during both components. The animal that reduced responding actually received significantly more shocks during avoidance than during punishment (19 per session in avoidance vs. 10 per session in punishment), while the other monkey received significantly more shocks during punishment than during avoidance (124 per session in punishment vs. 1 per session in avoidance). Only one of 
these monkeys actually received fewer shocks than the SCR-conditioned animals in Kimmel et al. (1979), while the other received more than twice as many shocks as the monkeys in Kimmel et al. Thus, there is little evidence to support the view that the acquisition of differentiation was poorer or more difficult in the autonomic case than in the skeletal case.

In the present study, discriminative stimuli made it easier for the monkey to acquire a pattern of SCR responding that achieved some degree of adaptive adjustment to the avoidance-punishment schedule employed. With the stimuli present, avoidancepunishment SCR differentiation came into existence relatively rapidly, overall SCR frequency levels did not increase appreciably, and shock frequency was moderate. With the stimuli absent, the acquisition of avoidance-punishment differentiation was retarded, overall SCR frequency was initially high and increased with training, and shock frequency was substantially elevated. Broadly speaking, the imposition of a multiple avoidance-punishment conditioning schedule contingent on an autonomic response, the SCR, has been shown in this study to be less traumatic to the monkey when exteroceptive discriminative stimuli that signal the avoidance and punishment contingencies are present. The monkey was able to acquire a significant degree of response differentiation, when discriminative stimuli were available, and generalized autonomic response frequency was not appreciably elevated. Without the stimuli, or with removal of the stimuli after conditioning with them, SCR differentiation was reduced, higher frequencies of shock were received, and higher general levels of SCR activation developed.

\section{REFERENCES}

Kimmet, H. D., Brennan, A. F., Mcleod, D. C., Raich, M. S., \& Schonfeld, L. I. Instrumental electrodermal conditioning in the monkey (Cebus albifrons): Acquisition and long-term retention. Animal Learning \& Behavior, 1979, 7, 447-451.

Kimmel, H. D., \& Bunns, R. A. The difference between conditioned tonic anxiety and conditioned phasic fear: Implications for behavior therapy. In I. G. Sarason C C. D. Spielberger (Eds.), Stress and anxiety (Vol. 4). Washington, D.C: Hemispheric, 1975.

RAich, M. S., \& KimmeL, H. D. Autonomic concomitants of discriminative avoidance and punishment training in the monkey. Pavlovian Journal of Biological Science, 1979, 14, 243-248.

\section{NOTES}

1. Subdermal needle electrodes were used by Kimmel et al. (1979) to pick up HR during initial training in the DSR group. The K\&G electrode substantially reduces movement artifacts in the HR data. These electrodes were donated by $K \& \mathrm{G}$ Healthcare Industries, Miami, Florida.

2. Figure 3 presents all four blocks of secondary training SCR frequency for comparison with their yoked controls.

(Manuscript received April 30, 1982; accepted for publication May 12, 1982.) 